\title{
Flood susceptibility mapping using a novel ensemble weights-of-evidence and support vector machine models in GIS
}

\begin{abstract}
Flood is one of the most devastating natural disasters that occur frequently in Terengganu, Malaysia. Recently, ensemble based techniques are getting extremely popular in flood modeling. In this paper, weights-of-evidence (WoE) model was utilized first, to assess the impact of classes of each conditioning factor on flooding through bivariate statistical analysis (BSA). Then, these factors were reclassified using the acquired weights and entered into the support vector machine (SVM) model to evaluate the correlation between flood occurrence and each conditioning factor. Through this integration, the weak point of WoE can be solved and the performance of the SVM will be enhanced. The spatial database included flood inventory, slope, stream power index (SPI), topographic wetness index (TWI), altitude, curvature, distance from the river, geology, rainfall, land use/cover (LULC), and soil type. Four kernel types of SVM (linear kernel (LN), polynomial kernel (PL), radial basis function kernel (RBF), and sigmoid kernel (SIG)) were used to investigate the performance of each kernel type. The efficiency of the new ensemble WoE and SVM method was tested using area under curve (AUC) which measured the prediction and success rates. The validation results proved the strength and efficiency of the ensemble method over the individual methods. The best results were obtained from RBF kernel when compared with the other kernel types. Success rate and prediction rate for ensemble WoE and RBF-SVM method were 96.48\% and $95.67 \%$ respectively. The proposed ensemble flood susceptibility mapping method could assist researchers and local governments in flood mitigation strategies.
\end{abstract}

Keyword: Flood susceptibility; Weights-of-evidence (WoE); Support vector machine (SVM); GIS; Remote sensing; Malaysia 